\title{
Lingual vibrotactile sensation magnitudes: Stimulus frequency effect
}

\author{
DONALD FUCCI and LINDA PETROSINO \\ School of Hearing and Speech Sciences, Ohio University, Athens, Ohio 45701
}

\begin{abstract}
The psychophysical method of magnitude production was used to obtain suprathreshold sensation magnitude functions at three frequencies $(100 \mathrm{~Hz}, 250 \mathrm{~Hz}, 400 \mathrm{~Hz})$ from the anterior midline portion of the tongue of 10 adult subjects. Results showed the slopes of the curves generated to be frequency dependent. The shallowest curve was obtained for the frequency of $250 \mathrm{~Hz}$, with somewhat steeper curves being derived for the frequencies of $100 \mathrm{~Hz}$ and $400 \mathrm{~Hz}$. An explanation of the frequency effect is attempted in terms of the possible neural receptor units involved in lingual suprathreshold vibrotactile stimulation.
\end{abstract}

In the late 1950 s, S. S. Stevens reported that a power function law successfully describes the relationship between subjective intensity and the magnitude of vibration applied to the neurophysiological mechanisms underlying cutaneous mechanoreception (Stevens, 1957). Stevens (1961) performed a series of studies relating subjective responses to stimulus intensities at the fingertip. More recently, several researchers have begun to systematically study the various parameters that can have an effect on suprathreshold vibrotactile power functions. Such parameters as psychophysical scaling method, the size of the skin contactor, the use of a rigid surface to surround the contactor, body site of stimulation, frequency at which scaling is performed, and signal duration have been found to have an effect on the magnitude functions obtained (Verrillo \& Capraro, 1975; Verrillo \& Chamberlain, 1972; Verrillo, Fraioli, \& Smith, 1969; Verrillo \& Smith, 1976; Zwislocki \& Goodman, 1980).

One of the parameters that has received particular attention is that concerned with the frequency at which scaling is performed. Stevens (1959) showed that the exponent of the power function for vibration can be altered as a function of stimulus frequency. He obtained flatter growth curves for $250 \mathrm{~Hz}$ than he did for $60 \mathrm{~Hz}$ at the fingertip. Verrillo et al. (1969) found no frequency effect on the power function exponent for a similar set of frequencies at the thenar eminence. Methods and experimental conditions were very different for these two studies, the two major differences being the site tested and the use of a rigid surround to confine the vibratory disturbance to the immediate area of the contactor. Verrillo employed the surround and Stevens did not. Verrillo and Capraro (1975) performed a more comprehensive study of the effect of stimulus frequency on subjective vibrotactile magnitude functions in which they used the method of magnitude estimation at $60 \mathrm{~Hz}$ and $250 \mathrm{~Hz}$ at the fingertip and thenar eminence, both with and without a rigid surface surrounding the contactor. They found that when the surround was in place, the slopes of the curves were independent of stimulus frequency. The slopes became frequency dependent when the surround was not used. The lower frequency of $60 \mathrm{~Hz}$ provided a steeper slope at both body sites tested.

The purpose of the present investigation was to provide further explanation of the frequency effect on subjective suprathreshold vibrotactile magnitude functions by obtaining power function curves at different frequencies from the human tongue. Exploration of the human tongue has been shown to be helpful in understanding the effect of frequency on vibrotactile threshold responses of cutaneous mechanoreceptors (Telage \& Warren, 1977; Verrillo, 1966).

\section{METHOD}

The vibrotactile instrumentation used in this study consisted of a stimulus unit and a measurement unit. The stimulus unit was composed of a sine-wave generator, an experimentercontrolled variable attenuator, a subject-controlled variable attenuator, two universal timers, an audio amplifier, a power amplifier, a preamplifier, and an electromagnetic minivibrator with a probe-contactor extension. The pulsed vibratory signal generated had a $50 \%$ duty cycle (on $500 \mathrm{msec}$ and off $500 \mathrm{msec}$ ), with a rise and decay time of $100 \mathrm{msec}$. The measurement unit included an accelerometer, a cathode follower, a microphone amplifier, and a voltmeter. A more detailed description of the vibrotactile equipment and procedures can be found in a stateof-the-art review by Fucci, Petrosino, Wallace, and Small (1982).

A group of 10 subjects having an age range of 22-32 years and a mean age of 24.3 years was employed in this study. All 10 subjects were treated in the same manner. Each subject was seated in an adjustable chair and asked to place his tongue up against the bottom of a rigidly mounted plastic disk. A hole in the center of the disk provided access for the probe-contactor extension of the vibrator to the anterior midline section of the dorsum of the tongue. The contactor on the end of the probe had an area of $.128 \mathrm{~cm}^{2}$, and there was a $1-\mathrm{mm}$ gap between the contactor and the rigidly mounted plastic disk. To maintain constant displacement of the contactor on the tongue for.all subjects, the vibrator assembly was lowered until a voltmeter recorded contact. The contactor was then lowered $1 \mathrm{~mm}$ further into the lingual surface.

The psychophysical method of magnitude production was 
used to establish suprathreshold magnitude functions for the tongue. This method was selected because it has been shown to be very stable (Zwislocki \& Goodman, 1980) and because it facilitates testing the tongue, in that the subject can respond by raising his hand rather than by providing a verbal numerical response (magnitude estimation) that would be difficult with the tongue in test position. The procedure used did not incorporate a standard modulus. A number of investigators have indicated that use of a standard reference can have a profound effect on the shape of the resulting magnitude function by interfering with the natural absolute magnitude scales that individuals have internalized (Zwislocki \& Goodman, 1980). The magnitude production task was performed by each subject at the frequencies of $100 \mathrm{~Hz}, 250 \mathrm{~Hz}$, and $400 \mathrm{~Hz}$. The order of frequency presentation was randomized across subjects. At each frequency, the subject was presented a random series of six numbers (5, $10,15,20,25,30)$, and he was asked to adjust the "magnitude" of the stimulus that he was feeling on his tongue to the number being presented. When he felt the task was completed, he was to raise his hand. The subject-controlled attenuator consisted of a smooth unmarked knob (120-dB variable potentiometer) having no visual or mechanical cues. The experimenter was in control of a master attenuator that could be used to vary the actual stimulus amplitude with respect to the position of the subject's attenuator knob, as a means of assurance that the subject was not developing external criteria to aid him in his task (Zwislocki \& Goodman, 1980). The numbers chosen for the magnitude production task were held to six because of adaptability and fatigue effects that appear to be more acute for the tongue than they are for some of the other mechanoreceptor systems that have been studied (Fucci \& Crary, 1979).

Three runs of the randomized series of six numbers were presented to each subject at each frequency. The first run was discarded, and the geometric means of the second and third runs were accepted as the produced amplitudes for that frequency (Verrillo et al., 1969). The results of the magnitude production task were recorded in millivolts and converted to displacement in decibels re 1-micron peak. Group data were generated by deriving geometric means of the magnitude production amplitudes across all subjects for each of the three frequencies.

\section{RESULTS AND DISCUSSION}

Figure 1 shows the results for the 10 subjects of the magnitude production task performed at the three frequencies of $100 \mathrm{~Hz}, 250 \mathrm{~Hz}$, and $400 \mathrm{~Hz}$. The data show that the mechanoreceptive mechanisms located within the anterior dorsal portion of the tongue can be used to produce suprathreshold magnitude functions at the three frequencies employed. The curves are very similar in shape to those reported by other investigators who did not use a standard reference, and the upper portions of the curves are compatible with Steven's power function law (Stevens, 1961; Verrillo \& Capraro, 1975; Verrillo \& Chamberlain, 1972; Verrillo et al., 1969; Zwislocki \& Goodman, 1980). The actual slopes of the upper portions of the curves for all three frequencies were higher than those typically reported in the literature for cutaneous receptor mechanisms. The slope values for $100 \mathrm{~Hz}, 250 \mathrm{~Hz}$, and $400 \mathrm{~Hz}$ were $1.58,1.45$, and 1.74 , respectively. Those values reported in the literature in terms of amplitude displacement are closer to or below unity (Stevens, 1961; Stevens, 1968;

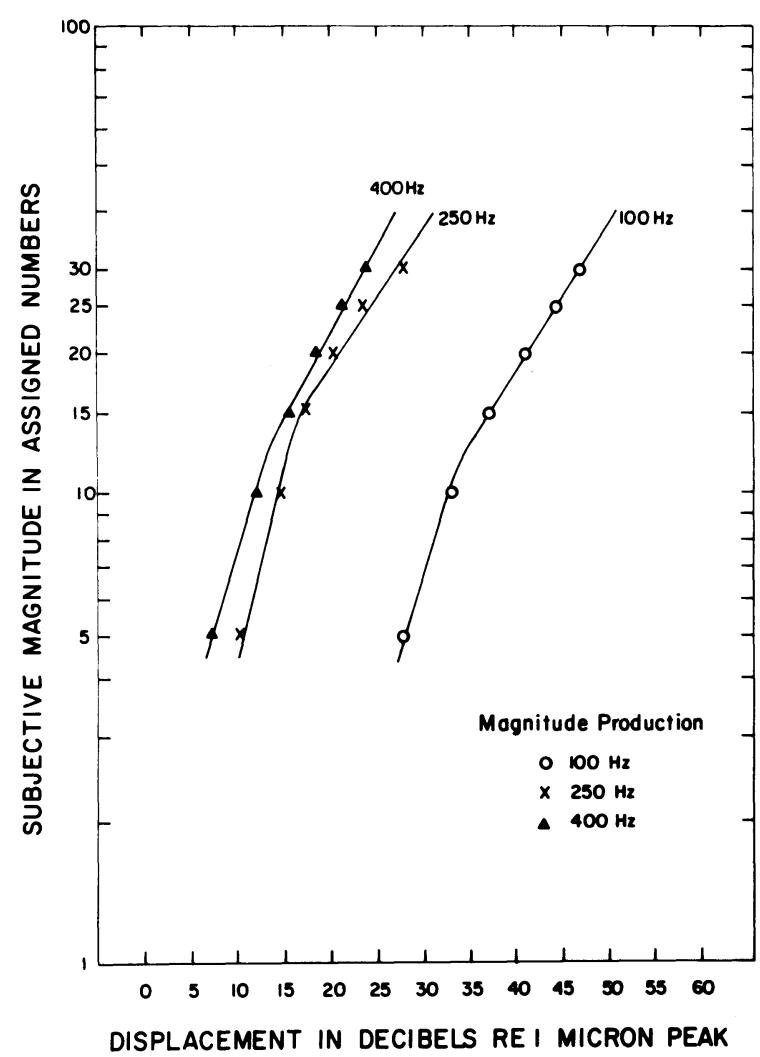

Figure 1. Magnitude production of lingual vibrotactile sensation (geometric means) as a function of displacement in decibels re 1-micron peak for a group of 10 subjects across three frequencies. Vibration frequencies were $100 \mathrm{~Hz}, 250 \mathrm{~Hz}$, and $400 \mathrm{~Hz}$.

Verrillo, 1979; Verrillo \& Chamberlain, 1972; Verrillo et al., 1969). The slope values for the three frequencies were also different with respect to each other. The shallowest curve (1.45) was obtained for the frequency of $250 \mathrm{~Hz}$, with somewhat steeper curves being derived for the other two frequencies.

A number of possible reasons for the steeper slope values for all three frequencies are reported in the literature. First, the method of magnitude production has been described by Stevens (1959) and Zwislocki and Goodman (1980) to produce slope values that are steeper than those produced by the method of magnitude estimation, which is commonly used in suprathreshold experimentation. Second, the size of the skin contactor has been shown by Verrillo and Chamberlain (1972) to have a significant effect on slope. The smaller the contactor, the steeper the slope will be. The smallest contactor used by Verrillo and Chamberlain (1972) was $.28 \mathrm{~cm}^{2}$, and the contactor used in this study $\left(.128 \mathrm{~cm}^{2}\right)$ was only half that size. Third, the use of a rigid surround is also reported to have an effect on curve slope in a manner similar to that of using a smaller contactor. In both instances, fewer sensory receptors are activated and the resulting slope is steeper (Verrillo 
\& Chamberlain, 1972). Fourth, the cutaneous location itself can have an effect on the slope of the curve (Stevens, 1961). A future controlled experiment in which lingual suprathreshold responses are compared to those of other body sites will have to be performed in order to determine the actual influence on slope that the tongue itself provides.

The reasons for slope differences between the frequencies are not entirely clear, especially when the use of a rigid surround is taken into account. One possible explanation might have to do with the nature of the tongue itself. The tongue, unlike the fingertip and thenar eminence, does not have bone lying immediately below the musculocutaneous surface. Consequently, vibrations applied to the lingual surface might be contained by the rigid surround on a horizontal plane, as they have been shown to be by other experimenters (Verrillo \& Chamberlain, 1972), but they might not be contained by the rigid surround on a vertical plane. In the absence of underlying bone, the vibrations could be traveling vertically down through the tongue and activating receptor units on the underlying ventral surface. This suggestion is made more plausible by the fact that the Pacinian corpuscle, a frequency-responsive receptor, is present on the ventral lingual surface and absent on the dorsal surface (Grossman \& Hattis, 1967; Winkelmann, 1960). It is a known fact that the Pacinian corpuscle is maximally sensitive to vibrations around $250 \mathrm{~Hz}$ and that this corpuscle can be excited over considerable distances by remote vibrations (Verrillo \& Capraro, 1975; Verrillo \& Chamberlain, 1972).

The acute sensitivity of the Pacinian corpuscle to $250-\mathrm{Hz}$ vibrations and its ability to be stimulated over large distances lead to the reasonable conclusion that, even though a rigid surround was employed around the contactor, vibrations could have traveled vertically downward through the tongue to activate the Pacinian corpuscles located in the ventral region. A greater number of receptors would be activated, then, for $250 \mathrm{~Hz}$ than for the other two frequencies, and the result would be the flatter subjective magnitude function actually found for that frequency. Further experimentation into cutaneous responses to vibration is necessary before more analytical explanations can be provided to explain the effect of frequency on the slopes of suprathreshold lingual vibrotactile magnitude functions.

\section{REFERENCES}

Fucci, D., \& Crary, M. Oral vibrotactile sensation and perception: State of the art. In N. J. Lass (Ed.), Speech and language: Advances in basic research and practice (Vol. 2). New York: Academic Press, 1979.

Fucci, D., Petrosino, L., Wallace, D., \& Small, L. H. Modification of instrumentation for research on lingual vibrotactile sensitivity: Elimination of the tongue clamping procedure. Review of Scientific Instruments, 1982, 53, 1294-1296.

Grossman, R. C., \& Hattis, B. F. Oral mucosal sensory innervation and sensory experience: $A$ review. In J. F. Bosma (Ed.), Symposium on oral sensation and perception. Springfield, Ill: Thomas, 1967.

Stevens, S. S. On the psychophysical law. Psychological Review, 1957, 64, 153-181.

Stevens, S. S. Tactile vibration: Dynamics of sensory intensity. Journal of Experimental Psychology, 1959, 57, 210-218.

STEvens, S. S. The psychophysics of sensory function. In W. A. Rosenblith (Ed.), Sensory communication. New York: Wiley, 1961.

Stevens, S. S. Tactile vibration: Change of exponent with frequency. Perception \& Psychophysics, 1968, 3, 223-228.

Telage, K. M., \& Warren, J. Spatial summation effects on lingual vibrotactile thresholds. Perceptual and Motor Skills, 1977, 44, 1179-1185.

Verrillo, R. T. Specificity of a cutaneous receptor. Perception \& Psychophysics, 1966, 1, 149-153.

VERRILLo, R. T. Comparison of vibrotactile threshold and suprathreshold responses in men and women. Perception \& Psychophysics, 1979, 26, 20-24.

Verrillo, R. T., \& Capraro, A. Effects of stimulus frequency on subjective vibrotactile magnitude functions. Perception \& Psychophysics, 1975, 17, 91-96.

Verrillo, R. T., \& Chamberlain, S. C. The effect of neural density and contactor surround on vibrotactile sensation magnitude. Perception \& Psychophysics, 1972, 11, 117-120.

Verrillo, R. T., Fraioli, A. J., \& Smith, R. L. Sensation magnitude of vibrotactile stimuli. Perception \& Psychophysics, 1969, 6, 366-372.

Verrillo, R. T., \& Smith, R. Effects of stimulus duration on vibrotactile sensation magnitude. Bulletin of the Psychonomic Society, 1976, 8, 112-114.

WinkelmanN, R. K. Nerve endings in normal and pathological skin. New York: Thomas, 1960.

Zwislocki, J., \& Goodman, D. Absolute scaling of sensory magnitudes: A validation. Perception \& Psychophysics, 1980, 28, 28-38.

(Received for publication September 1, 1982.) 